\title{
The Methods of Displaying DWF Image File on the Web Pages Accurately
}

\author{
Liu Qin \\ Wuhan University of Technology, Wuhan, China
}

\begin{abstract}
For various sectors ask to browse accurate positioning information's corresponding to the DWF image file of hot point on the map on the website platform, this paper proposes efficient and quick ways to archive browsing DWF image file online and position specific point precisely. After click the specific point on the map, the message of this point will be passed to the server services and front-end page gets returned information of this point's location on the DWF image file and the name of DWF file which will be showed on the web page. With the help of DWF Viewer ActiveX controls (short for ActiveX controls) from Autodesk Design View (short for ADR) software, the web page will display DWF image file dynamically according to different point on the map the users chosen. At last, the program will use returned message to display this DWF image file in this point as the center with an efficient way.
\end{abstract}

KEYWORDS: DWF image file; server service; ActiveX controls; positioning precisely

\section{INTRODUCTION}

With the development of computer technology, computer aided design (CAD) has been wirely used in construction, machinery, electronics and other industries. Especially in recent years, the rapid development of computer network communication technology, providing a new way of working for people so that the application of CAD technology will develop from the stand-alone version into the network direction (Li Ai Jun et al, 2004).

DWF (Design Web Format) format file, which is a kind of vector file, developed by Autodesk company as an open, safe drawing releasing format file, it is created by Autodesk application software (such as AutoCAD, Revit, and Inventor software) as the basis of graphics, it has the following advantages: the file size is smaller than DWG format file, which facilitates network transmission, distribution and carrying; DWF file is like the paper copy, suitable for viewing and marking (for plan approval), but can no longer editing and changing, which ensures the graphic information safe in the transmission process (Lin Sheng, 2014).

After DWF format file displays on the page dynamically, position the location you want to check precisely, this whole process brings more convenience for viewing the CAD files. Combining the navigation and position specific point on DWF with web technology, which makes it has higher extensi- bility, lays the foundation for the development of CAD technology in web terms, and provides a feasible way displaying CAD file in the project.

\section{DISPLAYING DWF IMAGE FILE ON THE WEB PAGE}

\subsection{Static displaying}

The method of browsing DWF image file introduced in this paper must be used on the local computer that is already installed ADR software, and object tag in HTML web page reference Autodesk DWF Viewer ActiveX controls (short for ActiveX controls) supported by Autodesk company to show DWF image file, so the displaying of DWF image file on web page is actually archived by ADR software on local computer. But ActiveX controls of this object tag needs classid property to call ADR software display DWF file, and the classid property is the constant reference of classid value in local computer registry (Wei Yong et al, 2009).

When the page is first loaded, the browser detects classid attribute that sets in object tag, then it prompts user to install ActiveX controls. After the installation of ActiveX controls is completely done, when the web page is loaded again, the browser will get the value of classid attribute and looks for the same value in the registry of local computer that corresponding to a software, when the native ADR 
software matchs the browser will call it to display DWF image file in the web page. But if the ActiveX controls is not installed on the browser, the whole process can't be made for lack of medium, so ActiveX controls is the key of linking web page with ADR software to display DWF image file and a tool to encapsulate ADR software into an object for the browser to call to display DWF image file, see our example on Fig. 1.

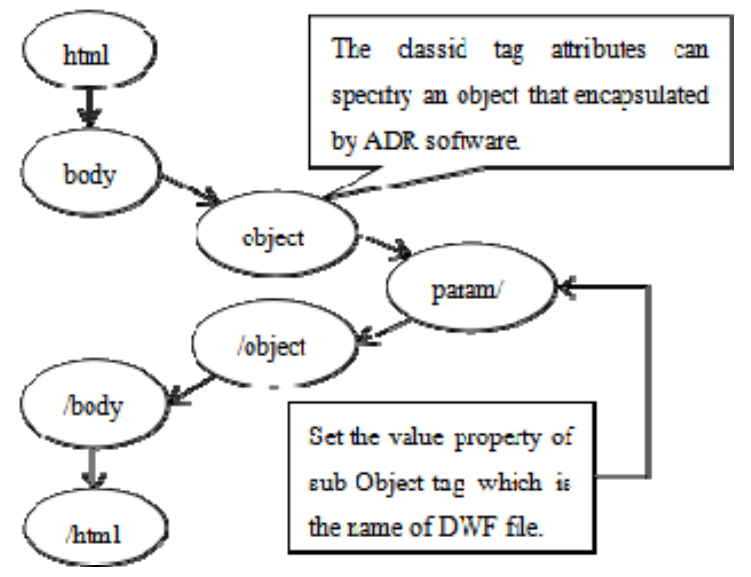

Fig. 1 Displaying statically

\subsection{Dynamic displaying}

DWF file can be displayed on the page normally statically, if the DWF file fixed in the HTML page all the same, but the hot point on the map is different, this static method can't display different DWF file corresponding to the point. So we must bind events for every hot point, the message of hot point will be sent to sever after click hot point. After check out the hot point's location information in DWF file by the database, the location information will be returned to the web page, then the object tag will use the information to display different DWF file. It's not working just modify the value of param sub-tag of object before the HTML file is completely loaded, which is to modify the name of DWF file which will be displayed in ActiveX controls, but it hasn't response after changing the attribute value of the controls. To solve this problem, we must insert the object controls element into DOM element before HTML page finish loading instead of changing the attributes value of object tag, see our example on Fig. 2.

\section{IMPLEMENTATION OF POSITIONING RANDOM LOCATION OF DWF FILE PRECISELY}

After DWF image file can be displayed on the web page correctly, we must move DWF file util the page is showing as center of the hot point that clicked on the map, so far to complete precise posi- tioning of hot point on the DWF image file. If you want to perform the operation to move the DWF file, it must be done after all DOM element of this page finish loading, but it just depend on the ADR API which supports the listener function On EndLoad Item to judge whether the DWF file finish loading completely, we must call function Wait For Section Loaded to wait all modules finish loading, then we can do relevant moving operation. Get the object element which attribute id is ADViewer, bind event On End Load Item (bstrItem Ty-pe, vData, vResult) for the element, the event will be triggered after DWF file finish loading. We must judge whether the event parameter bstrItem Type is DOCUMENT at the start of calling function, and get file node object collection called ADViewer. EComposite Viewer. Sections of showing software ADR of ActiveX controls, get every node object circularly and execute the method Wait For Section Loaded to wait all nodes finish loading if some nodes don't finish loading completely. After all nodes call function Section. Wait ForSection Loaded circularly, it means DWF file finish loading, we can continue to finish other precise positioning operation (Wang Jian Tao, 2005).

After the file has finished loading, it is necessary to complete the most important function of precise positioning, but there are many ways to achieve positioning, this article provides the following three methods to achieve.

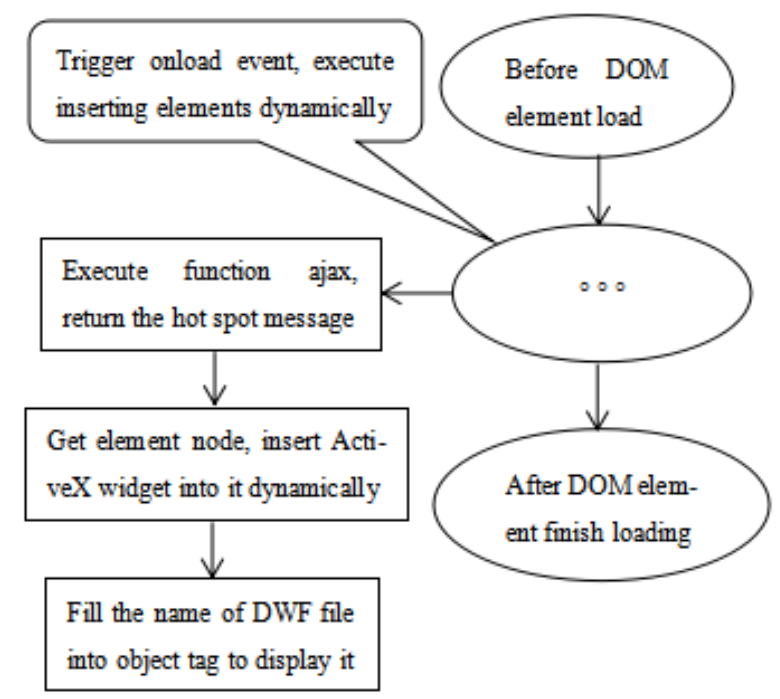

Fig. 2 Displaying dynamically

\subsection{Move positioning by keyboard scanning}

In daily life, when you skim the web page, it's hard to find important part to read if the page has a big length, but we usually click the page and hold down the direction key of keyboard util the important part is shown as the center of this page.

The principle of moving positioning by keyboard scanning is based on this, we move file to specific 
location by using the direction key of up and right; but if you want to operate the direction key in the program, you must rely JavaScript to get key object for using statement new ActiveXObject ("WScript.Shell"), after you get the object you can call the method SendKeys to simulate operation of up, bottom, left, right direction key, but the program must get the focus first, so you must execute function focus() of JavaScript to get focus on the DOM element before execute direction key. Of course, the success of key operation must rely on the previous work, we must take record times of executing up and right direction key to move DWF file from initial position to final, and then insert the records into the database, so when you click the specific point on the map, the moving data times will be returned from the server, then the program will execute key operation according to the times data to get the location in the DWF file.

However, when taking record of times to execute direction key, we often need many steps of key operation because of the big size of DWF file; we usually can't find the location of specific point in this case, the specific point may be outside the screen, so it's hard to take records. This will make previous work heavier and complex, we must reduce the view proportion to the level that we can see global view in order to solve this problem, so you can find the specific point on the DWF file accurately and reduce the times of key operation.

So we execute "Ctrl" and "-" combinations key to reduce the view proportion before executing up and right direction key operation when we take records. Meanwhile we must reduce the view proportion first at the actually positioning, because the moving distance by one step on the reduced view proportion is smaller than normal ones, this is why we must reduce the view proportion to the level of taking records at usual time, and then execute times of key operation at taking records, at last the whole process has no difference. But we must execute "Ctrl" and "0" combination key to restore the view proportion after finish all key operation at actually positioning, by doing this the DWF file can be shown normally, see our example on Fig. 3.

\subsection{Change steps of moving dynamically}

The principle of the above method is simple and clear, but it will have many drawback in actual practice. First of all, the whole process is relatively complex, it needs many steps of key operation to get the location of the specific location on the DWF file, so it's necessary to simplify these operations. Because of many steps of moving operation to get specific location, we must consider to change the distance of moving one step. According to API ADR supports, it provides a variable for JavaScript to alter the value of moving distance by one step, if we need thirty times of key operation to get to the specific location by the above method, then we can change the default moving variable ADViewer.EComposite Viewer. Pan Increment multiply it by thirty, so we can just move one step to get the same location. Similarly, we can change the value of reducing the view proportion by one step, then it can just need one step of "Ctrl" and "-"combination key to the view can be seen all. By checking ADR API for JavaScript, we know the default scaling parameter is fourty-three, the default moving step is thirty-three.

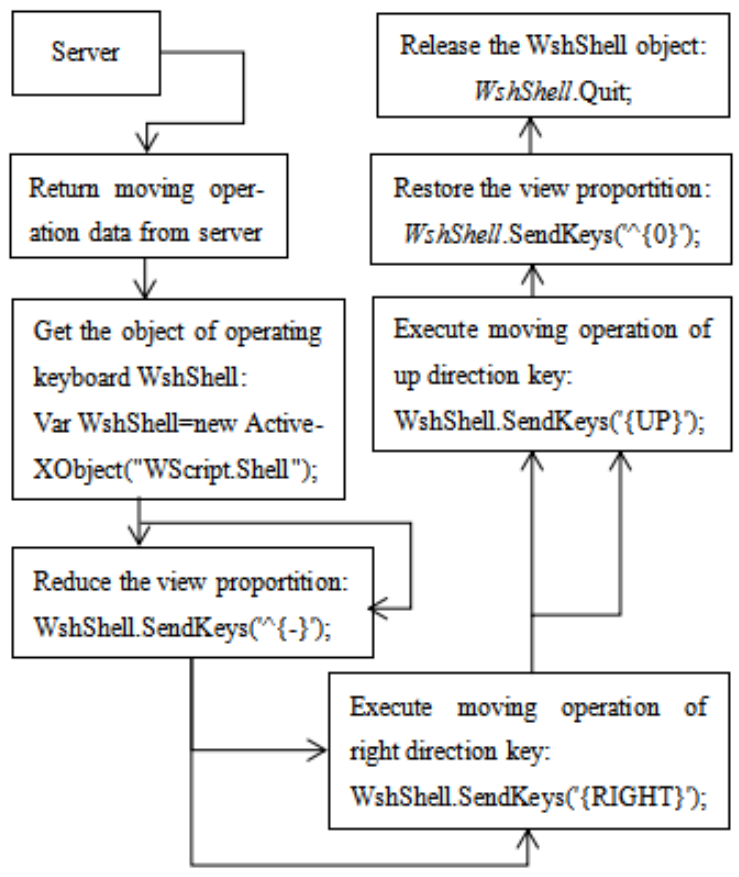

Fig. 3 Positioning by keyboard scanning

What we can find in above method, the whole positioning process needs to reduce the view proportion of "Ctrl" and "-" combination key, move operation of executing up and right direction key, restore the view proportion of "Ctrl" and " 0 " combination key, but if an operation is interrupted in this time, the follow-up operation cannot undertake, and continuous operations can't ensure be executed. So we must bind event for every key operation, then we can ensure execute next key operation before the last key operation is completely done. Of course, the ADR API provides handling event function for JavaScript to bind key listener event, then we can use the function to know when the last reducing operation finish so that we can continue to begin moving operation, see our example on Fig. 4.

\subsection{Set coordinate of view by calling native API method}

Those above methods are based on the keys operation, so we must keep focus on DWF file all the time in whole process, so we must also ensure nobody perform unnecessary operation like key or mouse 
operation, which has bad user experience and interactivity between user and web page. And when we reduce the view proportition of DWF file, it's easy to scale the entire browser, so that subsequent operation will be chaotic.

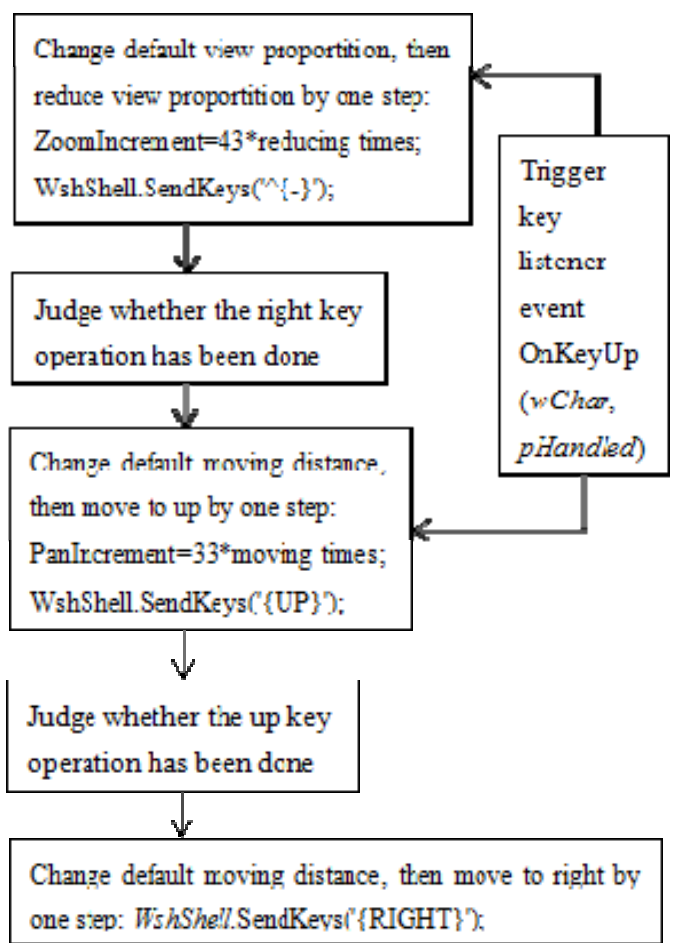

Fig. 4 Positioning by changing steps

Because of disadvantage of those above methods, we have to consider a better method to achieve fast and interference-free positioning. When we use software to search keyword in normal time, the software will highlight the keyword and show the view by center of it after input the keyword. Based on this idea, we must rely on the method ADR software supports for JavaScript to set the coordinate of view which is shown by center of the hot point that clicked on the map, but we must take records the coordinate of every hot point before actual positioning and insert the coordinate data into database from server. After check the API interface, we may find the API supports location coordinate message for each point on DWF file include up, bottom, left, right which states as JavaScript is ADViewer.Viewer.View.Top, ADViewer.Viewer.View.Bottom, ADViewer.Viewer.View.Left, AD Viewer.Viewer.View.Right, we can get the location coordinate message of every point by using these JavaScript variables, and the API also provides function ADViewer.Viewer.SetView (Top,Bottom,Left,Right) which can set the center of whole view and these parameters are the location coordinate message of each point.

Of course, the location coordinate message of each hot point must be recorded into database, then it will be given back to front page when you click the hot point on the map. It's very efficient to call native API method, it will finish positioning immediately after DWF file finish loading, and has no influence with users other operation, this method is this paper recommended.

\section{SUMMARY}

DWF format file is a common format of CAD drawing to publish on web page, and there are many complex methods to browse DWF file on the web page, but this paper offers a practical, efficient to fix problem. In this paper, the important part is how to display DWF file and how to display DWF file as the center of hot point that clicked on the map to achieve positioning. This technology brings profound significance for the CAD graphics displaying on the web page and other extending research, provides practical examples for flexible using of ActiveX controls and calling third-party software API for JavaScript, and provides a motivation for the development of the technology.

\section{REFERENCES}

Autodesk Design Review 2013 API Reference: http://usa.autodesk.com/adsk/servlet/index?siteID=123112\& id $=5801732$

Li Ai Jun, Xu Mao Feng.2004. Mechanical design and research, AutoCAD graphic posted online[J],20(5):45-46.

Lin Sheng.2014.The publishing and browsing of DWF files based on AutoCAD software, Equipment manufacturing technology [J], (9): 175-177.

Transform Point (JavaScript), Code example: http://usa.autodesk.com/adsk/servlet/index?siteID=123112\& id $=5801750$

Wang Jian Tao.2005.The Research and Implementation of GIServices based on Web Technology, Information Engineering University of the People's Liberation Army(ph.D. Thes-is):9-15.

Wei Yong Le, Chao Cai Xia.2009.Publishing and Browsing of DWF Figure File, Coal Mine Machinery [J],30(11):224-226. 